\title{
CULTIVO DE Brachiaria brizantha Stapf. cv. Marandu EM SOLUÇÃO NUTRITIVA COM OMISSÕES DE MACRONUTRIENTES
}

\author{
F.A. MONTEIRO ${ }^{1,3}$, A.K.B. RAMOS ${ }^{2,3}$, D.D. DE CARVALHO ${ }^{2,3}$, J.B.R. IE ABREU ${ }^{2,3}$, J.A.S. \\ DAIUB $^{2,3}$, J.E.P. DA SILVA ${ }^{2,3}$, W. NATALE, \\ 'Departamento de Química-ESALQ/USP, C.P. 9, CEP: 13418-900, Piracicaba-SP

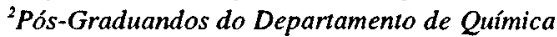 \\ ${ }^{2}$ Bolsistas do CNPq/CAPES
}

\begin{abstract}
RESUMO: Em experimento de casa de vegetação, cultivou-se Brachiaria brizantha Stapf. cv. Marandu em solução nutritiva, tendo como tratamentos a solução completa, as omissões individuais de $\mathrm{N}, \mathrm{P}, \mathrm{K}, \mathrm{Ca}, \mathrm{Mg}$, S e a testemunha (com omissão dos macronutrientes). Fez-se o acompanhamento da manifestação dos sintomas visuais de deficiência e, 50 dias após o transplante, avaliou-se a produção de matéria seca (MS), número de perfilhos (NP), a altura das plantas (H), bem como a composição mineral da parte aérea e das raízes. As omissões de $N$ e $P$ limitaram o crescimento, ao passo que a omissão de $K$ não afetou a produção de MS e NP. A omissāo de Ca não trouxe, para o período considerado, restrições na produção de MS, NP e H. Nas omissōes de Mg e S as plantas tiveram fraco desempenho, superando apenas os tratamentos onde foram omitidos o $\mathrm{N}$ e o $\mathrm{P}$. A composição mineral da parte aérea s6 apresentou diferenças acentuadas nos teores para os nutrientes omitidos. Sintomas visuais de deficiência foram observados para todas as omissōes, sendo os primeiros manifestados através da omissāo do $\mathrm{N}$ e do P e os últimos, através da omissão do Ca. São sugeridos estudos para melhor definição do balanço entre formas e fontes de nutrientes para a solução nutritiva quando utilizada no cultivo de plantas de Brachiaria brizantha cv. Marandu. Descritores: Brachiaria brizantha, nutrição mineral, macronutrientes, solução nutritiva, gramínea forrageira
\end{abstract}

\author{
GROWTII OF Brachiaria brizantha Stapf. Cv. MARANDU IN \\ NUTRIENT SOLUTION WITH MACRONUTRIENT OMISSIONS
}

\begin{abstract}
ARSTRACT: In a greenhouse experiment Brachiaria brizantha Stapf, cv. Marandu was grown in a nutrient solution. The treatments were a complete nutrient solution, nutrient solutions with the omissions of each macronutrient ( $N$, $\mathrm{P}, \mathrm{K}, \mathrm{Ca} . \mathrm{Mg}$ and $\mathrm{S}$ ) and a control (with the omission of all macronutrients). Plants were harvested 50 days after transplanting. Dry matter yield, tiller number and plant height were recorded, and nutrient concentrations in shoot and root were determined. Omissions of $N$ and $P$ were the most limiting for plant growth, whereas $K$ and $C$ a did not affect grass growth and tillering. $\mathrm{Mg}$ and $\mathrm{S}$ omissions resulted in significant decreases in the plant parameters. Great decreases in plant nutrient concentrations were verified in the treatments with nutrient omissions in solution. Visual symptoms of deficiencies were observed for each nutrient omission treatment, with those of $N$ and $P$ being the first and that of $\mathrm{Ca}$ being the latest shown by the plants. Studies on forms and sources of nutrients for nutrient solution to be used with this grass are suggested.
\end{abstract}

Key Words: Brachiaria brizantha, mineral nutrition, macronutrients, nutrient solution, forage grass

\section{INTRODUÇÃO}

Diversas são as espécies de gramíneas forrageiras tropicais que apresentam-se como opções para a formação de pastagens no Brasil. As do gênero Brachiaria têm-se firmado pela capacidade de adaptação às diversas condições ambientais e de manejo da pastagem.
A Brachiaria brizantha Stapf. cv. Marandu apareceu como mais uma opção para os pecuaristas, e pelas suas características agronômicas e índices zootécnicos permitidos já ocupa extensas áreas no Brasil Central. Como características, essa forrageira apresenta bom valor nutritivo, menor estacionalidade na produção, melhor relação folha/haste, e resistência a cigarri- 
nha das pastagens, quando comparada aos variedade do mesmo gênero (EMBRAPA,1985; ALCÂNTARA, 1986).

Apesar de bem adaptada às condições brasileiras e da crescente expressão, estudos sobre a nutrição mineral dessa braquiária ainda são escassos. O cultivar Marandu é recomendado para regiões com solos de média a alta fertilidade (EMBRAPA, 1985). Resultados experimentais mostraram boas respostas à adubação fosfatada, tolerância ao alumínio e pouca resposta à calagem, que seria recomendada como fonte de $\mathrm{Ca} \mathrm{e} \mathrm{Mg}$ (EMBRAPA, 1985 ; PEREIRA, 1987). Neste sentido, PREMAZZI (1991) observou máximas produções dessa forrageira quando a saturação por bases do solo atingiu $43 \%$.

Este trabalho teve por objetivo avaliar - comportamento da Brachiaria brizantha cv. Marandu frente a omissões individuais de macronutrientes, quando cultivada em solução nutritiva.

\section{MATERIAL E MÉTODOS}

O experimento foi conduzido em casade-vegetação na Escola Superior de Agricultura "Luiz de Queiroz", em Piracicaba,SP. Utilizaram-se vasos com capacidade de 3,6 litros, preenchidos com sílica, onde cultivou-se a Brachiaria brizantha cv. Marandu com a solução nutritiva descrita por SARRUGE (1975).

$O$ experimento contou com os seguintes tratamentos :

- Completo (N, P, K, Ca, Mg, S , B, Cl, Cu, Fe, Mn, Mo e $\mathrm{Zn}$ )

- Omissão de Nitrogênio (-N)

- Omissão de Fósforo (-P)

- Omissão de Potássio (-K)

- Omissão de Cálcio (-Ca)

- Omissão de Magnésio (-Mg)

- Omissão de Enxofre (-S)

- Testemunha (Sem os macronutrientes)

Os tratamentos foram dispostos em blocos completos, ao acaso, com três repetições. Efetuou-se rodízio semanal dos vasos, dentro de cada bloco experimental.

Nos tratamentos onde foram empregados $\mathrm{N}, \mathrm{P}, \mathrm{K}, \mathrm{Ca}, \mathrm{Mg}$ e $\mathrm{S}$, foram fornecidos nas doses respectivas de $210,31,234$, 200, 48 e $64 \mathrm{mg} / \mathrm{L}$. Todos os tratamentos receberam os micronutrientes $\mathrm{B}, \mathrm{Cl}, \mathrm{Cu}, \mathrm{Fe}, \mathrm{Mn}$, Mo e $\mathrm{Zn}$.

Para a quebra de dormência as sementes de Brachiaria brizantha cv. Marandu foram tratadas com ácido sulfúrico concentrado por três minutos, após o que foram lavadas com água corrente e depois com água deionizada, e postas para germinar em caixas do tipo 'gerbox' na câmara de germinação. Após dez dias foram transplantadas dez plântulas para cada vaso contendo sílica. Na primeira quinzena do experimento utilizou-se a solução diluída a 1/3 da solução nutritiva de cada tratamento. Nesse período realizaram-se desbastes periódicos, deixando-se cinco plantas por vaso.

Fez-se circular um litro da solução nutritiva pela sílica do vaso duas vezes durante o dia, e durante a noite a mesma era drenada, sendo efetuado o ajuste do volume da solução pela manhã. As soluções foram substituídas a cada quinze dias.

As plantas foram colhidas cinquenta dias após o transplante. O material foi lavado com água deionizada e separado em parte aérea e raízes, e colocado para secar em estufa a $70^{\circ} \mathrm{C}$ por um período de 72 horas. Após a secagem o material foi pesado e moído para ser analisado em laboratório, para determinações dos teores dos nutrientes na matéria seca da parte aérea e das raízes, conforme descrito por SARRUGE \& HAAG (1974).

O número de perfilhos e a altura das plantas foram observados por ocasião da colheita. Para a análise estatística dos resultados utilizou-se o teste de Tukey $(\boldsymbol{P}=0,05)$ para comparações múltiplas das médias, sendo que para a variável número de perfilhos adotou-se a transformação dos valores originais em $\sqrt{ }$.

\section{RESULTADOS E DISCUSSÃO}

Os resultados referentes à produção de matéria seca (MS) da parte aérea, raízes e total, bem como o número de perfilhos e a altura das plantas de Brachiaria brizantha cv. Marandu são apresentadas na TABELA 1 . Pode-se verificar que os tratamentos $-N$, - $P$ e testemunha foram os que mais limitaram o desenvolvimento das plantas $e$, consequentemente, a produção de MS, tanto na parte aérea como nas raízes, o que também se refletiu no número de perfilhos e na altura das plantas. Onde foi omitido o $\mathbf{N}$, inclusive na testemunha, as plantas alocaram maior parte da produção de biomassa para o sistema radicular. 
O tratamento com a omissão de $P$ apresentou, além de plantas raquíticas e sem perfilhos laterais (só tiveram um colmo de cada planta), elevada concentração de $\mathrm{N}$ na $\mathrm{MS}$ (TABELAS 2 e 3), quando comparado ao tratamento completo, caracterizando o efeito de concentração associado a plantas de crescimento limitado (JARREL \& BEVERLY,1981). Reduções drásticas na produção de MS e no perfilhamento, com as omissões de $N$ e $P$, também foram verificadas por FRANÇA \& HAAG (1985) em experimento com capim Tobiatã (Panicum maximum Jacq.).

Segundo WERNER (1986) o fósforo desempenha importante papel no desenvolvimento do sistema radicular e no perfilhamento da gramínea, sendo o $\mathrm{N}$ o principal constituinte das proteínas e participante ativo na síntese e composição da matéria orgânica que forma a estrutura vegetal. Por isso, os tratamentos com as omissões de $\mathrm{N}$ e $\mathrm{P}$ apresentaram sintomas visuais de deficiência logo nas primeiras semanas.

Os teores de $\mathrm{N}$ e $\mathrm{P}$ observados na parte aérea no tratamento completo situaram-se acima do comumente observado para a espécie (TABELA 2). COWARD-LORD et al. (1974) encontraram teores de $N$ de $1,47 \%$ na MS da parte aérea de plantas com 60 dias de crescimento após o corte e a adubação nitrogenada, ao passo que SOTOMAYORRÍOS et al. (1974) detectaram 1,74\% de N na MS da parte aérea aos 45 dias. Para o fósforo, ARROYO-AGUILÚ \& COWARD-LORD (1974) encontraram teores de $0,18 \%$ na MS.

A omissão de potássio não proporcionou redução significativa $(P>0,05)$ na produção de MS e no perfilhamento, em relação ao tratamento completo (TABELA 1). Tal fato não foi observado por FRANÇA \& HAAG (1985), onde a omissão de $K$ só não foi mais drástica do que nas omissóes de $\mathrm{N}$ e P. As TABELAS 2 e 3 mostram a porcentagem de $K$ na MS da parte aérea $(0,43 \%)$ e das raízes $(0,36 \%)$, que foram significativamente $(P<0,05)$ diferentes daquelas do tratamento completo, que apresentou valores de 2,80 e $2,50 \%$, respectivamente. PREMAZZI (1991) observou teor de 2,2\% de $K$ na MS da parte aérea nesse mesma braquiaria, enquanto ARROYO-AGUILÚ \& COWARDLORD (1974) encontraram, noutro cultivar, $2,38 \%$ de K. Sintomas visuais de deficiência de $\mathbf{K}$ foram observados a partir da terceira semana após o transplante nos vasos, com as plantas apresentando folhas mais estreitas e necrose nas margens das lâminas foliares. Sintomas semelhantes dessa carência foram relatados por WERNER (1971) para o capim-colonião (Panicum maximum Jacq.).

A omissão de magnésio resultou em redução na produção de matéria seca, superando apenas os tratamentos com a omissão de N, P e testemunha (TABELA 1). Sintomas visuais da deficiência de $\mathrm{Mg}$ ocorreram a partir da terceira semana após o transplante. As plantas apresentaram as lâminas foliares com clorose internerval, além de mostrarem-se prostradas nas últimas semanas. Sintomas similares também foram observados por WERNER (1971), em capim-colonião. Outro fato associado à omissão de $\mathbf{M g}$ foi a ocorrência de teores mais elevados dos nutrientes na matéria seca exceto $\mathrm{Mg}$, em relação ao tratamento completo (TABELAS 2 e 3). Tal fato pode estar associado à menor produção de MS nesse tratamento, que teria ocasionado a concentração dos nutrientes (JARREL $\&$ BEVERLY, 1981). No tratamento completo, o teor de $0,49 \%$ de $\mathbf{M g}$ observado na parte aérea chegou a ser duas vezes maior que o verificado por ARROYO-AGUILÚ \& COWARD-LORD (1974).

No tratamento em que se omitiu o enxofre, reduções significativas $(P<0,05)$ foram observadas para a produção de matéria seca, número de perfilhos e altura das plantas, quando comparado ao tratamento completo (TABELA 1). De acordo com ANDREW (1962) a deficiência de enxofre reduz a quantidade de nitrogênio convertida à forma orgânica, resultando em restrição ao crescimento da planta, por conta da proporção entre esses nutrientes nas proteínas. $O$ teor de $S$ na MS (parte aérea e raízes) também foi reduzido na omissão de $S$, sendo que nesse tratamento a relação $\mathrm{N}: \mathrm{S}$ foi maior $(19,7$ na parte aérea e 25,3 nas raízes) que no tratamento completo (14,1 na parte aérea e 9,5 nas raízes). Sintomas visuais de deficiência de $S$, com a verificação de clorose nas partes mais novas da planta, também foram observados.

O tratamento com omissão de cálcio apresentou a maior produção de MS, maior número de perfilhos e maior altura de plantas, inclusive em relação ao tratamento completo (TABELA 1). Cumpre salientar que a solução nutritiva de SARRUGE (1975), utilizada no experimento, apresenta diferenças nas formas de $\mathrm{N}$ fornecidas entre o tratamento completo e aquele com a omissão de Ca. No tratamento completo, o $\mathrm{N}$ é totalmente fornecido como nitrato $\left(\mathrm{NO}_{3}{ }^{-}\right)$, enquanto que no tratamento com omissão de $\mathrm{Ca}$, o nitrogênio 
$\varepsilon$, em parte, fornecido como amônio $\left(\mathrm{NH}_{4}{ }^{+}\right)$. A maioria das plantas absorve indistintamente nitrato e amônio. Todavia, fatores como idade, ambiente, espécie vegetal, bem como a disponibilidade no solo podem determinar a absorção preferencial de uma das formas. A forma amoniacal é incorporada pela planta praticamente sem gasto de energia, visto que já se encontra na forma reduzida, mas a utilização conjunta das duas formas de $\mathrm{N}$ pode levar a melhores desempenhos da planta (TISDALE et al., 1985).

LEYSHON et al. (1980) observaram que plantas de trigo puderam assimilar mais prontamente amônio que nitrato pois, nos primeiros estágios de desenvolvimento, o sistema nitratoredutase não estava em pleno funcionamento.

FRIED et al. (1965), trabalhando com duas fontes de $\mathrm{N}$, verificaram que raízes destacadas de arroz absorviam maiores quantidades do ín amônio e que o cálcio presente na solução nutritiva tendeu a inibir essa absorção. Tal fato pode explicar, em parte, o melhor desempenho, para o período considerado, das plantas submetidas à omissão de $\mathrm{Ca}$, pois o $\mathrm{N}$ como principal promotor de crescimento vegetativo não estaria sendo limitado.

Em se tratando de gramíneas tropicais, CIAT (1984) relatou melhores respostas à aplicação de nitrogênio na forma nítrica que na amoniacal, em trabalho com três espécies do gênero Brachiaria colhidas aos 90 dias. Entretanto, não foram utilizadas ambas as formas $\left(\mathrm{NO}_{3}{ }^{-}\right.$e $\left.\mathrm{NH}_{4}{ }^{+}\right)$ combinadas, e os níveis utilizados na solução eram bem inferiores aos do presente trabalho, o que poderia resultar em respostas diferentes. FERNANDES \& ROSSIELO (1986) expuseram que a utilização combinada das duas formas pode promover aproveitamento direto da forma amoniacal, com a formação de compostos nitrogenados como proteinas estruturais e enzimas, as quais poderiam estimular a ação do sistema nitratoredutase, uma vez que o nitrato acumulado no "pool" substrato não induziria a ação enzimática. $\mathrm{O}$ amônio poderia aumentar a absorção do nitrato, dando maior atividade ao sistema enzimático, o que é perfeitamente possível e favorecido com a troca das soluções durante o ensaio, a exemplo do trabalho de FERNANDES et al. (1978) onde doses baixas de amônio eram intercaladas com doses mais elevadas de nitrato. TISDALE et al. (1985) descreveram ainda que a absorção na forma amoniacal pode estimular a absorção de $\mathrm{P}$ e $\mathrm{S}$, reduzindo a do $\mathrm{Ca}, \mathrm{Mg}$ e $\mathrm{K}$.

Com a omissão de cálcio foi observado um aumento na concentração de $S$ e uma diminuição na concentração de $\mathbf{M g}$, em relação ao tratamento completo (TABELA 2). Na última semana do experimento foram observados sintomas visuais característicos da deficiência de $\mathrm{Ca}$, com as plantas mostrando folhas mal formadas, com bordos crestados e com os ápices foliares afinados e retorcidos.

TABELA 1 - Produção de matéria seca (g/vaso), número de perfilhos e altura de plantas de $B$. brizantha cv. Marandu cultivada em solução nutritiva, 50 dias após o transplante. Médias de três repetições.

\begin{tabular}{|c|c|c|c|c|c|}
\hline \multirow[b]{2}{*}{ TRATAMENTO } & \multicolumn{2}{|c|}{ Matéria seca (g/vaso) } & \multirow[b]{2}{*}{ total } & \multirow{2}{*}{$\begin{array}{c}N^{0} \text { perfilhos } \\
\text { por vaso }\end{array}$} & \multirow{2}{*}{$\begin{array}{l}\text { altura } \\
\text { (cm) }\end{array}$} \\
\hline & Parte aérea & raízes & & & \\
\hline Completo & $14,27 \mathrm{~b}$ & $5,90 \mathrm{~b}$ & $20,17 \mathrm{~b}$ & $25 \mathrm{~b}$ & $84 a$ \\
\hline Omissão de $\mathrm{N}$ & $0,08 \mathrm{~d}$ & $0,10 \mathrm{e}$ & $0,19 \mathrm{e}$ & $5 \mathrm{~d}$ & $4 d$ \\
\hline Omissão de P & $0,82 d$ & $0,47 \mathrm{e}$ & $1,29 \mathrm{e}$ & $5 \mathrm{~d}$ & $25 \mathrm{c}$ \\
\hline Omissão de K & $11,17 \mathrm{~b}$ & $4,93 \mathrm{~b}$ & $16,10 \mathrm{bc}$ & $25 \mathrm{~b}$ & $65 \mathrm{~b}$ \\
\hline Omissão de $\mathrm{Ca}$ & 23,91 a & 0,65 a & $34,56 \mathrm{a}$ & $34 a$ & $83 a$ \\
\hline Omissão de $\mathbf{M g}$ & $7,79 \mathrm{c}$ & $1,78 \mathrm{~d}$ & $9,57 \mathrm{~d}$ & $17 \mathrm{c}$ & $65 \mathrm{~b}$ \\
\hline Omissão de $\mathbf{S}$ & $7,78 \mathrm{c}$ & $3,52 \mathrm{bc}$ & $11,30 \mathrm{~cd}$ & $17 \mathrm{c}$ & $59 \mathrm{~b}$ \\
\hline Testemunha & $0,07 \mathrm{~d}$ & $0,14 \mathrm{e}$ & $0,21 \mathrm{e}$ & $5 \mathrm{~d}$ & $4 d$ \\
\hline Teste $F$ & $* *$ & $* *$ & $* *$ & $* *$ & $* *$ \\
\hline DMS $(0,05)$ & 4,45 & 1,97 & 6,36 & 7 & 12,40 \\
\hline CV (\%) & 18,8 & 19,9 & 18,9 & 14,7 & 8,9 \\
\hline
\end{tabular}

1 - 5 plantas por vaso; ${ }^{2}$ - Médias originais. Análises com a transformação $\sqrt{ } \mathrm{x} ; * *$ - Significativo $(P<0,01)$ Médias seguidas de letras diferentes, dentro de cada coluna, diferem estatisticamente entre si pelo teste de Tukey $(P<0,05)$. 
TABELA 2 - Concentração de nutrientes na matéria seca da parte aérea de Brachiaria brizantha cv. Marandu cultivada em solução nutritiva, 50 dias após o transplante. Médias de três repetições.

\begin{tabular}{|c|c|c|c|c|c|c|c|c|c|c|}
\hline TRATAMENTO & $\mathrm{N}$ & $\mathbf{P}$ & K & $\mathrm{Ca}$ & Mg & $\mathbf{S}$ & $\mathrm{Cu}$ & $\mathrm{Fe}$ & $\mathrm{Zn}$ & $\mathrm{Mn}$ \\
\hline & & & & \multicolumn{3}{|c|}{ \% - - } & \multicolumn{4}{|c|}{ mg/kg - } \\
\hline Completo & $2,68 \mathrm{a}$ & $0,24 c$ & $2,80 \mathrm{bc}$ & $0,85 \mathrm{bc}$ & $0,49 \mathrm{a}$ & $0,19 b$ & $4,00 \mathrm{ab}$ & $80 \mathrm{ab}$ & $14,3 \mathrm{c}$ & 34de \\
\hline Omissão de $\mathrm{N}$ & $0,85 \mathrm{c}$ & n.d. & n.d. & n.d. & n.d. & n.d. & n.d. & n.d. & n.d. & n.d. \\
\hline Omissão de P & $2,76 \mathrm{a}$ & $0,04 d$ & $3,34 a b$ & $1,57 \mathrm{a}$ & $0,34 a b$ & n.d. & $4,33 \mathrm{ab}$ & $68 \mathrm{bc}$ & $16,0 \mathrm{bc}$ & $18 \mathrm{e}$ \\
\hline Omissão de K & $2,54 a$ & $0,37 \mathrm{~b}$ & $0,43 d$ & $1,00 \mathrm{~b}$ & $0,42 a b$ & $0,17 b$ & $4,33 a b$ & $66 \mathrm{bc}$ & $16,0 \mathrm{bc}$ & $62 \mathrm{~cd}$ \\
\hline Omissão de Ca & $1,97 \mathrm{~b}$ & $0,27 b c$ & $2,17 \mathrm{c}$ & $0,09 d$ & $0,36 a b$ & $0,25 \mathrm{a}$ & $6,67 a$ & $105 \mathrm{a}$ & $22,3 a b$ & $92 \mathrm{bc}$ \\
\hline Omissão de Mg & $2,45 \mathrm{a}$ & $0,50 \mathrm{a}$ & $4,03 a$ & $0,86 \mathrm{bc}$ & $0,02 c$ & $0,22 \mathrm{ab}$ & $6,67 \mathrm{a}$ & $75 a b c$ & $27,3 a$ & $161 \mathrm{a}$ \\
\hline Omissão de S & $1,97 \mathrm{~b}$ & $0,25 \mathrm{c}$ & $2,30 \mathrm{c}$ & $0,71 \mathrm{c}$ & $0,26 \mathrm{~b}$ & $0,10 \mathrm{c}$ & $3,33 \mathrm{~b}$ & $43 c$ & $13,3 \mathrm{c}$ & $22 \mathrm{e}$ \\
\hline Testemunha & $0,80 \mathrm{c}$ & n.d. & n.d. & n.d. & n.d. & n.d. & n.d. & n.d. & n.d. & n.d. \\
\hline Teste $F$ & $* *$ & $* *$ & $* *$ & $* *$ & $* *$ & $* *$ & $* *$ & $* *$ & $* *$ & ** \\
\hline $\operatorname{DMS}(0,05)$ & 0,44 & 0,10 & 1,01 & 0,16 & 0,21 & 0,05 & 2,67 & 36,5 & 7,9 & 37 \\
\hline CV $(\%)$ & 7,7 & 13,2 & 14,3 & 6,9 & 24,5 & 11,2 & 19,3 & 17,7 & 15,3 & 20,3 \\
\hline
\end{tabular}

n.d. - Não determinado por insuficiência de material.

** - Significativo $(P<0,01)$

Médias seguidas de letras diferentes, dentro de cada coluna, diferem estatisticamente entre si pelo teste de Tukey $(P<0,05)$.

TABELA 3 - Concentrações de nutrientes na matéria seca das raízes de Brachiaria brizantha cv. Marandu cultivada em solução nutritiva, 50 dias após o transplante. Médias de três repetições.

\begin{tabular}{|c|c|c|c|c|c|c|c|c|c|c|}
\hline \multicolumn{2}{|c|}{ TRATAMENTO N } & $\mathbf{P}$ & $\mathrm{K}$ & $\mathrm{Ca}$ & $\mathbf{M g}$ & $\mathbf{s}$ & $\mathrm{Cu}$ & $\mathrm{Fe}$ & $\mathrm{Zn}$ & $\mathrm{Mn}$ \\
\hline & & & & \multicolumn{3}{|c|}{ \% - - } & \multicolumn{4}{|c|}{ mg/kg } \\
\hline Completo & $2,27 \mathrm{a}$ & $0,17 b$ & $2,50 \mathrm{~b}$ & $0,46 b c$ & $0,35 \mathrm{ab}$ & $0,24 b$ & $6 \mathrm{~b}$ & $239 \mathrm{~b}$ & $20 \mathrm{c}$ & $36 c$ \\
\hline Omissão de N & $0,64 b$ & n.d. & n.d. & n.d. & n.d. & n.d. & n.d. & n.d. & n.d. & n.d. \\
\hline Omissão de P & $2,06 \mathrm{a}$ & $0,05 \mathrm{c}$ & $2,86 a b$ & $0,43 b c$ & $0,45 \mathrm{a}$ & n.d. & $7 b$ & $230 \mathrm{~b}$ & $23 \mathrm{c}$ & $36 \mathrm{c}$ \\
\hline Omissão de K & $2,24 \mathrm{a}$ & $0,19 b$ & $0,36 \mathrm{~d}$ & $0,45 b c$ & $0,27 b$ & $0,28 \mathrm{ab}$ & $4 b$ & $140 b$ & $28 \mathrm{c}$ & $52 \mathrm{c}$ \\
\hline Omissão de Ca & $1,61 \mathrm{ab}$ & $0,15 b$ & $1,48 \mathrm{c}$ & $0,08 \mathrm{~d}$ & $0,24 b$ & $0,37 \mathrm{a}$ & $15 \mathrm{~b}$ & $466 a$ & $42 \mathrm{~b}$ & $207 \mathrm{~b}$ \\
\hline Omissão de $\mathrm{Mg}$ & $1,64 \mathrm{a}$ & $0,35 \mathrm{a}$ & $3,60 \mathrm{a}$ & $0,70 \mathrm{a}$ & $0,04 c$ & $0,30 a b$ & $38 \mathrm{a}$ & $478 \mathrm{a}$ & $107 a$ & $490 \mathrm{a}$ \\
\hline Omissão de S & $2,53 \mathrm{a}$ & $0,17 \mathrm{~b}$ & $3,21 \mathrm{ab}$ & $0,32 \mathrm{c}$ & $0,33 \mathrm{a}$ & $0,10 \mathrm{c}$ & $6 \mathrm{~b}$ & $160 \mathrm{~b}$ & $19 \mathrm{c}$ & $27 \mathrm{c}$ \\
\hline Testemunha & $0,59 b$ & n.d. & n.d. & n.d. & n.d. & n.d. & n.d. & n.d. & n.d. & n.d. \\
\hline Teste $F$ & $* *$ & ** & ** & ** & ** & $* *$ & ** & ** & ** & ** \\
\hline DMS $(0,05)$ & 0,98 & 0,04 & 0,95 & 0,16 & 0,15 & 0,09 & 15 & 149 & 25 & 102 \\
\hline CV (\%) & 20,0 & 9,6 & 14,4 & 14,7 & 20,0 & 13,6 & 31,2 & 18,5 & 22,1 & 25,6 \\
\hline
\end{tabular}

n.d. - Não determinado por insuficiência de material.

** - Significativo $(P<0,01)$

Médias seguidas de letras diferentes, dentro de cada coluna, diferem estatisticamente entre si pelo teste de Tukey $(P<0,05)$.

Os teores dos micronutrientes na matéria seca da parte aérea e nas raízes da Brachiaria brizantha cv. Marandu são apresentados nas TABELAS 2 e 3, respectivamente.

PREMAZZI (1991) observou teores de Fe na MS da parte aérea muito próximos $(94,28$ $\mathrm{mg} / \mathrm{kg}$ ) aos observados neste experimento para o tratamento completo $(80 \mathrm{mg} / \mathrm{kg})$. Por outro lado os valores de manganês obtidos por PREMAZZI (1991) foram muito mais elevados $(114,4 \mathrm{mg} / \mathrm{kg})$ que os encontrados neste experimento $(35 \mathrm{mg} / \mathrm{kg})$. Tal fato, provavelmente deveu-se à utilização de 
solo naquele experimento. GALLO et al. (1974) com amostras de tecidos da parte aérea de $B$. decumbens, também encontraram teores $\mathrm{de} \mathrm{Cu}, \mathrm{Fe}$, $\mathrm{Mn}$ e $\mathrm{Zn}$, superiores ao obtido neste estudo, exceto na omissão de $\mathrm{Mg}$, para o $\mathrm{Fe}$ e $\mathbf{M n}$.

Com relação à solução nutritiva, SARRUGE (1975) ao propô-la para experimentos envolvendo deficiências, relatou que a sua escolha depende de fatores ligados à solução, ao clima e à espécie. Este fato pode ser evidenciado neste experimento onde a omissão de $\mathrm{Ca}$, para o período considerado, resultou em incremento na produção de matéria seca. Nesse sentido, CIAT (1986) relatou a limitação de algumas técnicas para diagnóstico bionutricional em germoplasmas, onde a eficiência relativa da solução nutritiva de HOAGLAND \& ARNON (1950) foi superior para $B$. decumbens quando comparada à $B$. milliformis. Assim, parece oportuno sugerir estudos sobre fontes e formas de nutrientes para adequação da solução de SARRUGE (1975), quando utilizada $\mathrm{em}$ experimentos com $B$. brizantha Stapf. $\mathrm{cv}$. Marandu.

\section{CONCLUSÕES}

- As omissões de N, P, Mg e $S$ limitaram o crescimento e a produção de matéria seca da parte aérea e das raízes da forrageira.

- A omissão de cálcio possibilitou produção de matéria seca superior à do tratamento completo, o que foi parcialmente atribuído às fontes de nitrogênio utilizadas na composição da solução nutritiva.

- Os nutrientes omitidos apresentaram-se em teores mais baixos nos tecidos vegetais e sintomas visuais de deficiência foram ohtidos nessa braquiária, para cada um dos macronutrientes estudados.

- Mais estudos relacionados à composição da solução nutritiva de SARRUGE, envolvendo Brachiaria brizantha Stapf. cv. Marandu, são sugeridos.

\section{REFERÊNCIAS BIBLIOGRÅFICAS}

ALCÂNTARA, P.B. Origem das Brachiarias e suas características morfológicas de interesse forrageiro. In: ENCONTRO PARA DISCUSSĀO SOBRE CAPINS DO GÊNERO BRACHIARIA, 1., Nova Odessa, 1986. Anais... Nova Odessa: Instituto de Zootecnia, 1987. p.1-18.
ANDREW, C.S. Influence of nutrition on nitrogen fixation and growth of legumes. In: COMMONWELTH SCIENTIFIC INDUSTRIAL RESEARCH ORGANIZATION. A review of nitrogen in the tropics with particular reference to pasture. A symposium. Farnham Royal, CSIRO, Melbourne, 1962. p.130-146.

ARROYO-AGUILÚ, J.A.; COWARD-LORD, J. Mineral composition of 10 tropical forage grasses in Puerto Rico. The Journal of the University of Puerto Rico, Río Piedras, v.58, n.4, p.426-436, 1974.

CIAT. Programa de pastos tropicales. Informe CIAT, Cali, p.251-256, 1984.

COWARD-LORD, J.; ARROYO-AGUILÚ, J.A.; GARCÍA-MOLINARI, O. Proximate nutrient composition of 10 tropical forage grasses. The Journal of the University of Puerto Rico, Río Piedras, v.58, n.3, p.305-311, 1974.

EMPRESA BRASILEIRA DE PESQUISA AGROPECUÁRIA. Centro Nacional de Pesquisa de Gado de Corte. Brachiaria brizantha v. Marandu. Campo Grande, 1985. 31p. (EMBRAPA/CNPGC. Documentos, 21).

FERNANDES, M.S.; FERREIRA, M.B.; FREIRE, L.R. Efeitos da interação $\mathrm{N}-\mathrm{NO}_{3}$ e $\mathrm{N}-\mathrm{NH}_{4}$ na atividade da nitrato redutase e acumulação de $\mathrm{N}$-protéico em Brachiaria sp. Turrialba, San José, v.28, n.3, p.187191,1978

FERNANDES, M.S.; ROSSIELO, R.O.P. Aspectos do metabolismo e utilização do nitrogênio em gramíneas tropicais. In: SIMPÓSIO SOBRE CALAGEM E ADUBAÇÃO DE PASTAGENS, 1., Nova Odessa, 1985. Anais... Piracicaba: POTAFOS, 1986. p.93-123.

FRANÇA, A.F.S; HAAG, H.P. Nutrição mineral de gramíneas tropicais. I. Carências nutricionais de capim tobiatã (Panicum maximum Jacq.). Anais da Escola Superior de Agricultura "Luiz de Queiroz", Piracicaba, v.42, n.1, p.83-96, 1985.

FRIED, M.; ZSOLDOS, F.; VOSE, P.B.; SHATOKHIN, I.L. Characterizing the $\mathrm{NO}_{3}$ and $\mathrm{NH}_{4}$ uptake process of rice roots by use of ${ }^{15} \mathrm{~N}$ labelled $\mathrm{NH}_{4} \mathrm{NH}_{3}$. Physiologia Plantarum, Kobenhain, v. 18 , n.2, p.313-320, 1965.

GALLO, J.R.; HIROCE, R.; BATAGLIA, O.C.; FURLANI, P.R.; FURLANI, A.M.C.; MATTOS, H.B.; FONSECA, M.P. Composição química inorgânica de forrageiras do Estado de São Paulo. Boletim de Indústria Animal, Nova Odessa, v.31, n.1, p.115-137, 1974. 
HOAGLAND, D.R.; ARNON, D.I. The watter-culture method for growing plants without soil. Circular. California Agriculture Experimental Station. Berkeley, n.347, 1950.

JARREL, W.M; BEVERLY, R.B. The dilution effect in plant nutrition studies. Advances in Agronomy, New York, v.34, n.1, p.197-224, 1981.

LEYSHON, A.J.; CAMPBELL, C.A.; WARDER, F.G. Comparison of the effect of $\mathrm{NO}_{3}$ and $\mathrm{NH}_{4}-\mathrm{N}$ on growth and yield components of manitou spring wheat and conquest barley. Canadian Journal of Plant Science, Ottawa, v.60, n.4, p.1063-1070, 1980.

PERERA, J.P. Adubação de capins do gênero Brachiaria. In: ENCONTRO PARA DISCUSSĀO SOBRE CAPINS DO GÊNERO BRACHIARIA, 1. Nova Odessa, 1986. Anais... Nova Odessa: Instituto de Zootecnia, 1987, p.117-196.

PREMAZZI, L.M. Saturação por bases como critério para recomendação de calagem em cinco forrageiras tropicais. Piracicaba, 1991. 215p. Dissertação (Mestrado) - Escola Superior de Agricultura "Luiz de Queiroz", Universidade de São Paulo.

SARRUGE, J.R. Soluçōes nutritivas. Summa Phytopatologica, Jaguariúna, v.1, n.3, p.231-233, 1975.
SARRUGE, J.R.; HAAG, H.P. Análises qứmicas em plantas. Piracicaba: ESALQ, Departamento de Química, 1974. 56p.

SOTOMAYOR-RÍOS, A.; JULIÁ, F.J.; ARROYOAGUILU, J.A. Effects of harvest intervals on the yield and composition of 10 forage grasses. The Journal of the University of Puerto Rico, Río Piedras, v.58, n.4, p.448-455, 1974.

TISDALE, S.L.; BEATON, J.D.; NELSON, W.L. Soil fertility and fertilizers. 4.ed. New York: Mac Millan, 1985. 754p.

WERNER, J.C. Adubação de pastagens. Nova Odessa, Instituto de Zootecnia, 1986. 49p. (1Z. Boletim Técnico, 18).

WERNER, J.C. Estudo sobre a nutrição mineral de alguns capins tropicais. Piracicaba, 1971. 95p. Tese (Doutorado) - Escola Superior de Agricultura "Luiz de Queiroz", Universidade de São Paulo.

Entregue para publicação em 30.05.94

Aceito para publicação em 17.08.94 